\title{
Article \\ Investigation of Temperature-Dependent Magnetic Properties and Coefficient of Thermal Expansion in Invar Alloys
}

\author{
Lin Huang ${ }^{1, *} \mathbb{C}$, Yongjian Zhou ${ }^{1}$, Tingwen Guo ${ }^{1}$, Dong Han ${ }^{2}$, Yu Gu ${ }^{2}$, Cheng Song ${ }^{1}$ and Feng Pan ${ }^{1}$ \\ 1 Key Laboratory of Advanced Materials (MOE), School of Materials Science and Engineering, Tsinghua \\ University, Beijing 100084, China; zhouyj18@mails.tsinghua.edu.cn (Y.Z.); \\ gtw18@mails.tsinghua.edu.cn (T.G.); songcheng@mail.tsinghua.edu.cn (C.S.); \\ panf@mail.tsinghua.edu.cn (F.P.) \\ 2 Shanxi Taigang Stainless Steel Co., Ltd., Taiyuan 030003, China; handong@tisco.com.cn (D.H.); \\ guyu01@tisco.com.cn (Y.G.) \\ * Correspondence: lhuang2020@mail.tsinghua.edu.cn; Tel.: +86-185-1328-5122
}

Citation: Huang, L.; Zhou, Y.; Guo, T.; Han, D.; Gu, Y.; Song, C.; Pan, F. Investigation of TemperatureDependent Magnetic Properties and Coefficient of Thermal Expansion in Invar Alloys. Materials 2022, 15, 1504 https://doi.org/10.3390/ ma15041504

Academic Editor: Carmine Maletta

Received: 10 December 2021

Accepted: 15 February 2022

Published: 17 February 2022

Publisher's Note: MDPI stays neutral with regard to jurisdictional claims in published maps and institutional affiliations.

Copyright: (C) 2022 by the authors. Licensee MDPI, Basel, Switzerland. This article is an open access article distributed under the terms and conditions of the Creative Commons Attribution (CC BY) license (https:// creativecommons.org/licenses/by/ $4.0 /)$.

\begin{abstract}
Invar Fe-Ni alloy is a prominent $\mathrm{Ni}$ steel alloy with a low coefficient of thermal expansion around room temperature. We investigate the correlation between magnetic properties and thermal expansion in cold-drawn $\mathrm{Fe}-36 \mathrm{Ni}$ wires with different heat treatment conditions, where the annealing parameters with furnace cooling (cooling from the annealing temperature of 300, 400, 500, 600, 700, 800,900 , and $1000^{\circ} \mathrm{C}$ ) are used. The variation trend of magnetic properties is consistent with that of thermal expansion for all samples, where the maximum appears at $600{ }^{\circ} \mathrm{C}$-treated sample and $400{ }^{\circ} \mathrm{C}$ shows the minimum. The domain size and the area of domain walls determine the total energy of the domain wall, and the total energy directly determines the size of magnetostriction, which is closely related to the coefficient of thermal expansion. Also, the differential thermal analysis (DTA) shows endothermic and exothermic reactions represent crystalline transitions, which could possibly cause the abrupt change of magnetic properties and thermal expansion coefficient of materials. The results indicate that there is a certain relation between thermal expansion and magnetic properties. Besides the fundamental significance, our work provides an Invar alloy with a low coefficient of thermal expansion for practical use.
\end{abstract}

Keywords: invar alloy; coefficient of thermal expansion; domain structure; magnetostriction

\section{Introduction}

In 1897, Guillaume found a very low coefficient of expansion $\left(0.877 \times 10^{-6} /{ }^{\circ} \mathrm{C}\right)$ in $36 \% \mathrm{Ni}-\mathrm{Fe}$ alloys, which did not completely conform to the normal thermal expansion law. It was an abnormal thermal expansion phenomenon and was called Invar alloy [1,2]. The factors affecting the thermal expansion coefficient of Invar alloys mainly include alloy composition, grain size, defect content, and precipitation. The Invar concentration $35.7 \% \mathrm{Ni}-\mathrm{Fe}$ (mass fraction) alloy shows the lowest thermal expansion coefficient, and the thermal expansion coefficient increases significantly regardless of the change of $\mathrm{Ni}$ or Fe content on both sides of the composition. Therefore, accurate control of Ni content is the key to obtain the Invar alloy [3]. It also has been reported that the internal defects of $\mathrm{Ni}-\mathrm{Fe}$ alloys increase and the density decreases due to cold machining, which destroys the degree of short-range atomic ordering and affects the spontaneous magnetization and magnetostriction system of the alloys. Finally, the thermal expansion coefficient of the alloys is reduced, and even becomes negative [4]. Invar alloys also exhibit particular magnetic properties such as sudden deviation of spontaneous magnetization, the large high susceptibility, strong pressure-dependence of the Curie temperature $\left(T_{c}\right)$, and an easy demagnetization [5-7]. The phenomena above are generally considered as Invar effects. Invar alloys are widely used in aeronautics and astronautics, motor valves, seismic creep, 
precision instruments, etc. [8-10]. Thus, Invar alloys have attracted extensive attention with increasing interest [11-13].

In general, a common material reflects a nature of thermal expansion and cold contraction, showing a normally large coefficient of thermal expansion [14]. Differently, Invar alloys always show an abnormally small coefficient of thermal expansion, which is commonly explained by the specific changes in their micro-structure [15], the formation of a short-range or long-range order, and of a modulated structure [16-18]. Alternatively, the low coefficient of thermal expansion of Invar alloys may also be caused by the fact that the normal lattice variation is compensated by the robust spontaneous volume magnetostriction in a wide temperature range $[9,19,20]$. However, how these magnetic properties systematically affect $\alpha$ is still unknown. The experiments below investigate the correlation between magnetic properties and the coefficient of thermal expansion in cold-drawn $\mathrm{Fe}-$ $36 \mathrm{Ni}$ wires with different heat treatment conditions. From our results, we found that the trend of thermal expansion is consistent with that of saturation magnetization and energy density of magnetic domain determines the size of magnetostriction with different heat treatment conditions. Moreover, the differential thermal analysis (DTA) shows endothermic and exothermic reactions represent crystalline transitions, which indicates the abrupt change of magnetic properties and thermal expansion coefficient of materials.

\section{Experimental Section}

The materials used in this work are cold-drawn Invar Fe-36Ni alloy wires prepared by Shanxi Taigang Stainless Steel Co., Ltd., Shanxi, China, with a diameter range of 2-4 mm. The chemical composition of raw sample is shown in Table 1. Based on the composition, it is a typical Fe-36Ni alloy (hereafter referred to as raw sample). Invar alloy wires were annealed at different temperatures (T) of 300 (sample T300), 400 (T400), 500 (T500), 600 (T600), 700 (T700), 800 (T800), 900 (T900), and $1000^{\circ} \mathrm{C}$ (T1000) for $2 \mathrm{~h}$ and then underwent furnace cooling. Magnetic properties were characterized by vibrating sample magnetometer (VSM) (LakeShore 8604 Westerville, OH, USA), and magnetic force microscope (MFM). The differential thermal analysis (DTA) was measured by thermogravimetric-differential thermal analysis (No.STA499F3 NETZSCH-Gerätebau GmbH, Selb, Germany). The coefficients of thermal expansion were measured by a thermal analysis system (No.DIL805ADT, New Castle, DE, USA), and the coefficient of thermal expansion value $\alpha$ was the average value of measured temperature range form $20-100{ }^{\circ} \mathrm{C}$ (heating start temperature was $15{ }^{\circ} \mathrm{C}$ and end temperature was $120^{\circ} \mathrm{C}$ ), the temperature ramp was $5^{\circ} \mathrm{C} / \mathrm{min}$. Moreover, we used cylindrical samples with a diameter of $4 \mathrm{~mm}$ and length of $10 \mathrm{~mm}$; each sample was repeated three times to extract the coefficient of thermal expansion.

Table 1. Composition of the cold-drawn Invar Fe-Ni alloy wire with $w t \% \geq 0.01 \%$.

\begin{tabular}{ccccccc}
\hline $\mathbf{C}$ & $\mathbf{S i}$ & $\mathbf{M n}$ & $\mathbf{A l}$ & $\mathbf{C u}$ & $\mathbf{N i}$ & $\mathbf{F e}$ \\
\hline 0.02 & 0.15 & 0.29 & 0.016 & 0.01 & 35.71 & 63.79 \\
\hline
\end{tabular}

\section{Results and Discussion}

\subsection{VSM Studies}

In the following experiments, we mainly focus on the influence of magnetic properties of Invar alloy on thermal expansion coefficient. After heat treatment at different temperatures, the relationship between magnetic variation on magnetostriction and thermal expansion coefficient is obtained. The magnetic properties of Invar alloy wires with different heat treatment conditions are investigated. The perpendicular magnetic hysteresis loops ( $M-H$ loops) in Figure 1 are measured at room temperature. We repeat the measurement with three samples to extract the saturation magnetization $M_{s}$. All of the $M-H$ loops demonstrate strong soft magnetic properties with small coercive field $\left(H_{c}\right)$ (smaller than $15 \mathrm{Oe}$ ). $H_{c}$ is extracted from the $M-H$ loops and samples of annealing temperature at $400{ }^{\circ} \mathrm{C}$ and $600{ }^{\circ} \mathrm{C}$ show almost the same $H_{c}$ and higher than others. However, the 
saturation magnetization $\left(M_{S}\right)$ with different annealing temperatures shows a significantly different trend, which affects the variation trend of thermal expansion coefficient, as we will explain later.

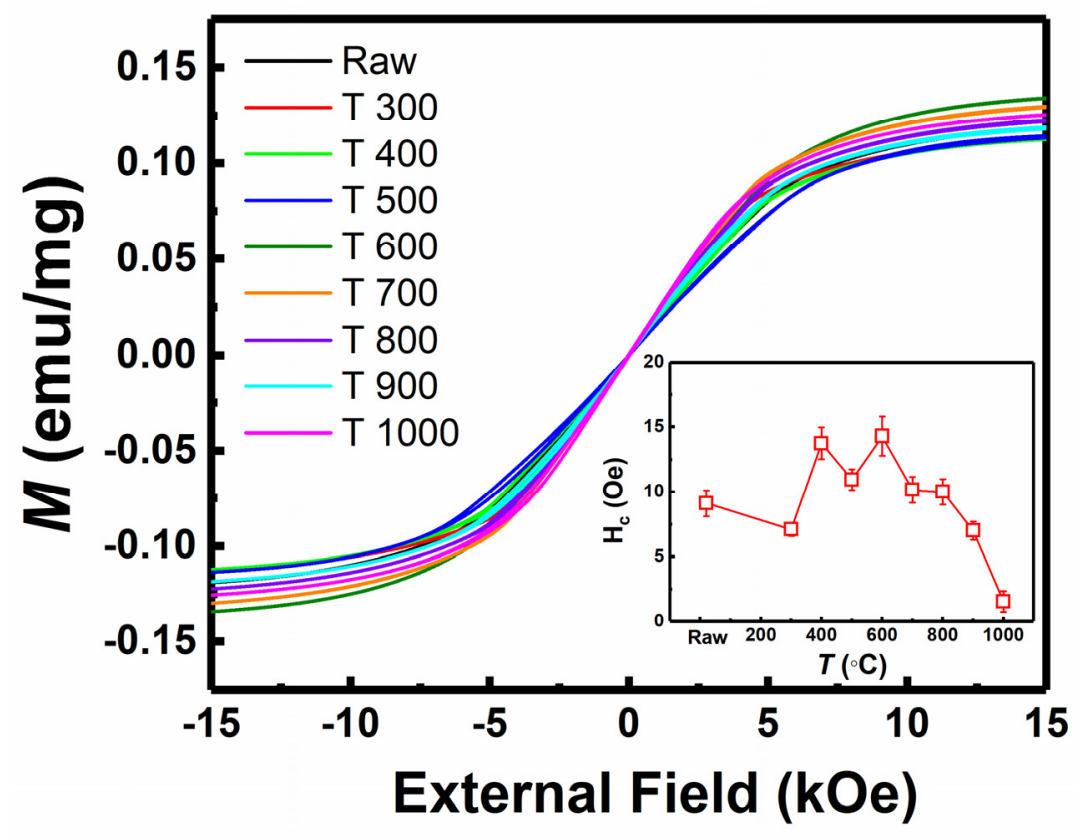

Figure 1. $M-H$ loops of Invar alloy wires with various heat treatment conditions (raw, T300, T400, T500, T600, T700, T800, T900, T1000), respectively. And $H_{\mathcal{C}}$ as a function of heat treatment temperature (T) extracted from $M-H$ loops is shown in the inset.

\subsection{Comparison of Thermal Expansion Coefficient $\alpha$ and Saturation Magnetization $M_{S}$}

The coefficient of thermal expansion $\alpha$ as a function of heat treatment temperature is shown in Figure 2a. Wherein, the coefficient of thermal expansion value $\alpha$ is the average value of measured temperature range form $20-100{ }^{\circ} \mathrm{C}$ (heating start temperature is $15^{\circ} \mathrm{C}$ and end temperature is $120^{\circ} \mathrm{C}$ ), the temperature ramp is $5^{\circ} \mathrm{C} / \mathrm{min}$. Moreover, we used the cylindrical samples with a diameter of $4 \mathrm{~mm}$ and length of $10 \mathrm{~mm}$; each sample was repeated three times to extract the coefficient of thermal expansion. In particular, with the increasing of annealing temperature, the change of thermal expansion coefficient does not show a linear trend, it is similar with the trend of saturation magnetization $M_{S}$ (Figure $2 \mathrm{~b})$. At the heat treatment, condition T400 shows the lowest $M_{\mathcal{S}}(0.1125 \mathrm{emu} / \mathrm{mg})$ and coefficient of thermal expansion value $\alpha\left(0.73 \times 10^{-6} /{ }^{\circ} \mathrm{C}\right)$; T600 shows the highest $M_{S}(0.1342 \mathrm{emu} / \mathrm{mg})$ and the coefficient of thermal expansion value $\alpha\left(1.75 \times 10^{-6} /{ }^{\circ} \mathrm{C}\right)$. The details of Invar alloy wires about different heat treatment conditions, XRD phases, $M_{s}$, and $\alpha$ are summarized in Table 2. It is worth noting that the $\alpha$ value of T600 is much higher than T400, which indicates the magnetic properties play a role for the coefficient of thermal expansion for Invar alloy wires. It is indicated that the thermal expansion value $\alpha$ is proportional to the $M_{s}$, and to the $\alpha \propto \Delta \mathrm{L} / \mathrm{L}$ value, which is as well as spontaneous volume magnetostriction $\lambda$. The fact that we can estimate that the spontaneous volume magnetosriction $\lambda$ is proportional to $M_{S}$ is consistent with the research, as Ref. [21] showed. The spontaneous volume magnetostriction $\lambda$ is proportional to the square of the magnetization $M^{2}$ as: $\lambda={ }_{k} C^{\text {band }} \mathrm{M}^{2}$, where $\mathrm{C}^{\text {band }}$ is the magnetovolume couping constant [21]. 
(a)

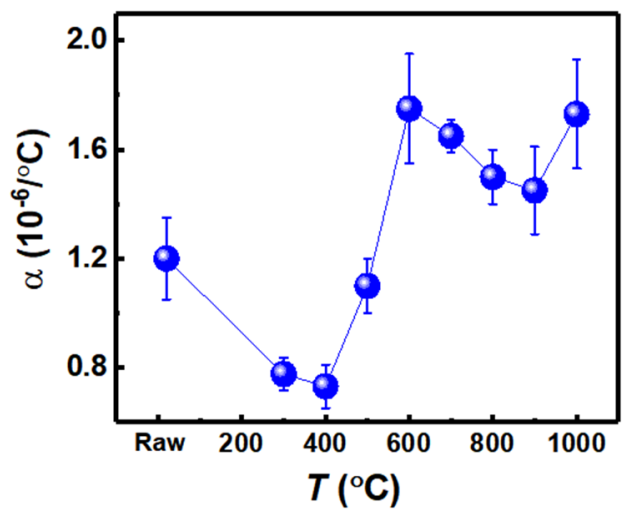

(b)

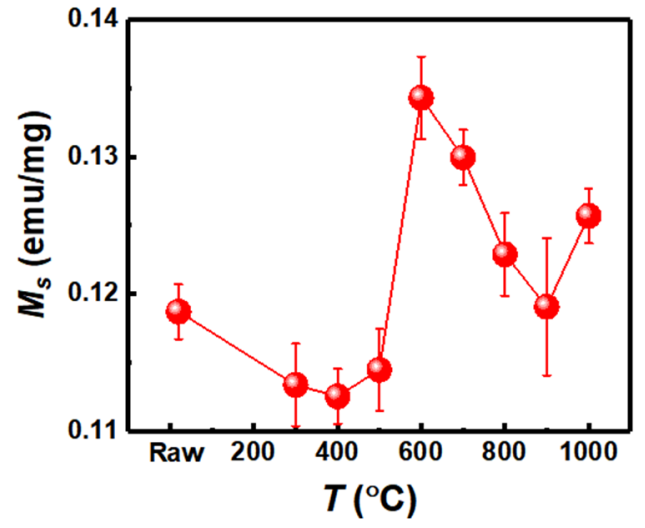

Figure 2. (a) The coefficient of thermal expansion $(\alpha)$ as the function of the heat treatment temperature (T). (b) Saturation magnetization $M_{S}$ as a function of heat treatment temperature $(T)$ extracted from $M-H$ loops.

Table 2. Different heat treatment conditions, XRD phase, saturation magnetization $\left(M_{S}\right)$, and coefficient of thermal expansion $(\alpha)$.

\begin{tabular}{cccc}
\hline Heat Treatment Conditions & XRD Phase & $\boldsymbol{M}_{\boldsymbol{s}}(\mathbf{e m u} / \mathbf{m g})$ & $\boldsymbol{\alpha}\left(\mathbf{1 0}-\mathbf{6} / \mathbf{C}^{\mathbf{c}}\right)$ \\
\hline Raw & fcc & 0.1187 & 1.2 \\
\hline T300 & fcc & 0.1133 & 0.77 \\
\hline T400 & fcc & 0.1125 & 0.73 \\
\hline T500 & fcc & 0.1144 & 1.1 \\
\hline T600 & fcc & 0.1342 & 1.75 \\
\hline T700 & fcc & 0.1299 & 1.65 \\
\hline T800 & fcc & 0.1228 & 1.5 \\
\hline T900 & fcc & 0.1190 & 1.45 \\
\hline T1000 & fcc & 0.1256 & 1.73 \\
\hline
\end{tabular}

\subsection{MFM Studies}

Furthermore, the magnetic domain shows the direct evidence which verifies the effect of magnetic properties on coefficient of thermal expansion. MFM images measured at room temperature are shown in Figure 3, raw, T300, T400, T500, T600, T700, T800, T900, T1000 correspond to Figure $3 \mathrm{a}-\mathrm{i}$, respectively. However, as the heat treatment temperature varies, the total energy $f=\varepsilon S(T)$ [22] of the domain walls changes, where $\varepsilon$ is the domain wall energy density and $S(T)$ is the domain wall area of samples with annealing temperature $T$. Note that MFM could only detect out of plane magnetization, thus all black and white magnetic domains are 180 degrees, which are antiparallel to each other. In the equilibrium structure, the relationship between the domain wall energy density $\varepsilon$ and domain wall 
bending degree $\mathrm{R}$ is: $\varepsilon \propto \mathrm{R}$. Among all samples, the domain wall area of the T400 sample is the largest and the degree of bending is highest, while that of T600 is the smallest. It indicates the highest total energy of domain walls $f$ and domain wall energy density $\varepsilon$ with T400, and lowest with T600. The relationship between the initial magnetic susceptibility $\chi_{i}$ of Invar alloys and the energy density of the domain walls is $\chi_{i} \propto \frac{\mathrm{M}_{\mathrm{s}}^{2}}{\varepsilon}$ [22], which results in a minimum $\chi_{i}$ value of T400 and maximum of T600. According to the above analysis, the change of material magnetostriction $\lambda$ can be expressed as: $\lambda \propto \frac{1}{\chi_{i}} \propto \varepsilon$. Additionally, it can be estimated that the area of domain $S$ is inversely proportional to the $\lambda$, and the results shown in Figure 2 are also supporting this opinion. Therefore, according to previous discussions, the material magnetostriction $\lambda$ of samples can be defined as $\lambda_{\mathrm{T} 400}>\lambda_{\text {(Raw, T300, T500, T700, T800, T900, T1000) }}>\lambda_{\mathrm{T} 600}$. T400 obtains the highest magnetostriction and T600 the lowest. Magnetostriction is a change in volume, size or shape caused by a change in the magnetization state of a ferromagnet. Therefore, it is of practical significance to utilize and control this change in the study of Invar alloys. When bending of the magnetic domain is large, the magnetostriction is relatively large, then the magnetic expansion can effectively eliminate the normal lattice expansion, resulting in a very low coefficient of thermal expansion. Conversely, a high coefficient of thermal expansion can be obtained if magnetostriction is weak. Therefore, the highest coefficient of thermal expansion $\alpha$ is shown in T600 and lowest $\alpha$ in T400. The above well explains the relationship between the change of thermal expansion coefficient and magnetic properties.
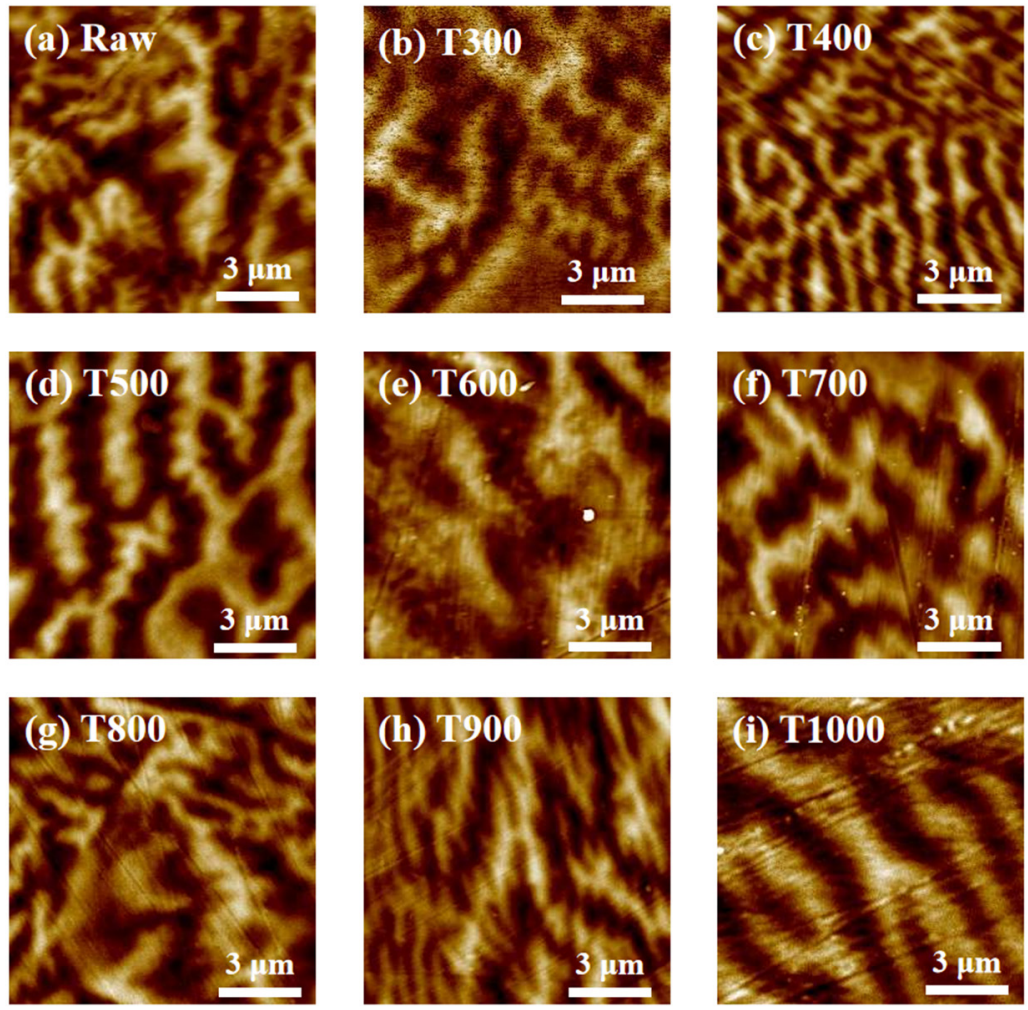

Figure 3. Magnetic domain images of raw (a), T300 (b), T400 (c), T500 (d), T600 (e), T700 (f), T800 (g), T900 (h), and T1000 (i), at room temperature.

\subsection{DTA Studies}

Moreover, the differential thermal analysis (DTA) measured by thermogravimetricdifferential thermal analysis (TG-DTA) in an inert atmosphere of argon, at the temperature range from $50{ }^{\circ} \mathrm{C}$ to $1000{ }^{\circ} \mathrm{C}$, is plotted in Figure 4e. It shows the change of crystallization in invar alloy samples with increasing temperature. There is an obvious endothermic peak near $600{ }^{\circ} \mathrm{C}$, and the phenomenon of endothermic peak is accompanied by the crystal transformation of alloy material. There is also a relatively small endothermic peak 
near $400{ }^{\circ} \mathrm{C}$, and a clear exothermic peak at $900{ }^{\circ} \mathrm{C}$, which is an exothermic reaction. Both endothermic and exothermic reactions represent the crystalline state of samples. After holding at $400{ }^{\circ} \mathrm{C}$ for $2 \mathrm{~h}$ annealing (Figure $4 \mathrm{~b}$ ), the recovery process was basically completed, that is, the storage energy caused by deformation was released. With the increase of annealing temperature $600{ }^{\circ} \mathrm{C}$ (Figure 4c), the grains grew bigger, indicating their recrystallization. When the annealing temperature is increased to $900{ }^{\circ} \mathrm{C}$ (Figure $4 \mathrm{~d}$ ), the grain coarsening is obvious. It is known that when the shape variable is constant, the higher the temperature, the larger the grain grows. We noted that the endothermic and exothermic peaks correspond to the changes of trends of saturation magnetization and thermal expansion.

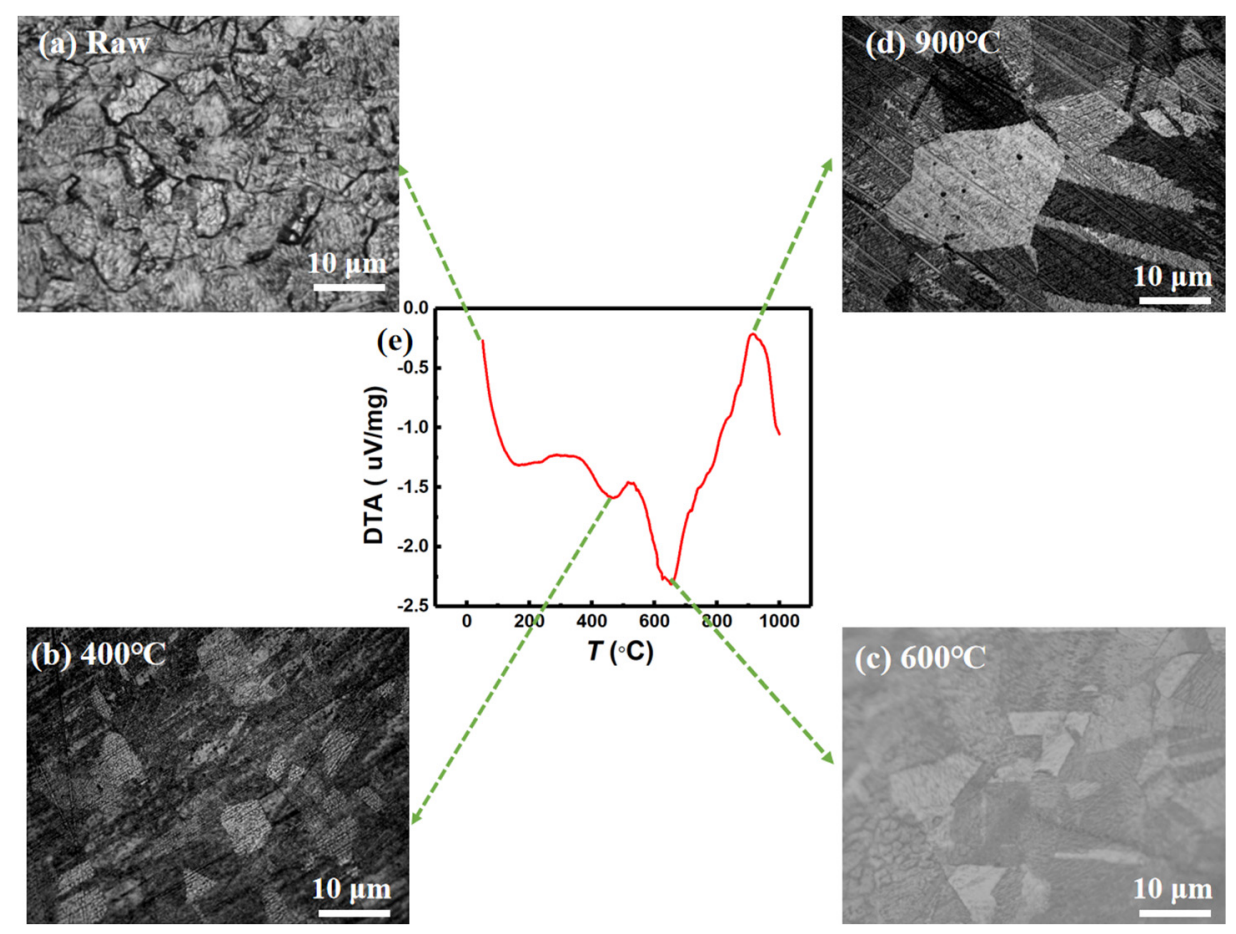

Figure 4. Micrograin structure of samples treated with different heat treatment processes: (a) raw, (b) $400{ }^{\circ} \mathrm{C}$, (c) $600{ }^{\circ} \mathrm{C}$, and (d) $900{ }^{\circ} \mathrm{C}$. (e) Differential thermal analysis (DTA) as the function of temperature (range from $50{ }^{\circ} \mathrm{C}$ to $1000{ }^{\circ} \mathrm{C}$ ).

From the above experimental results, it is known more clearly that the change of magnetic properties of the Invar alloy has a definite influence on the change of magnetostriction and thermal expansion. The coefficient of thermal expansion is proportional to magnetostriction $\lambda$ and the square of the magnetization $M^{2}$, but inversely proportional to the area of the magnetic domain $S$. This obvious change in magnetic properties allows us to provide more methods and a more comprehensive perspective on the size of the thermal expansion coefficient.

\section{Conclusions}

We have investigated the effect of magnetic properties on the coefficient of thermal expansion with different heat treatment conditions for cold-drawn Fe-36Ni Invar alloy wires. By comparing the magnetic properties of the samples, the variation trend of all samples is consistent with that of the thermal expansion coefficient, T600 shows the maximum and T400 shows the minimum. The domain size and the area of domain walls determines the total energy of the domain wall, and the size of the energy directly determines the size of the magnetostriction, which is closely related to the coefficient of thermal expansion. Magnetostriction can effectively eliminate the normal lattice expansion, thus resulting in a low coefficient of thermal expansion. Moreover, the differential thermal analysis (DTA) shows 
endothermic and exothermic reactions represent crystalline transitions, which indicates the abrupt change of magnetic properties and thermal expansion coefficient of materials. The results indicate that there is a certain relation between thermal expansion and magnetic properties. Besides the fundamental significance, our work provides an Invar alloy with a low coefficient of thermal expansion for practical use. This obvious change in magnetic properties allows us to provide more methods and a more comprehensive perspective for estimating the thermal expansion coefficient.

By observing the relationship between the coefficient of thermal expansion and magnetic properties, we can know that magnetic property is one of the factors affecting the change of thermal expansion coefficient. It is believed that magnetostriction has a certain effect on the thermal expansion coefficient, which cancels out the normal lattice contraction, resulting in an almost zero expansion rate at room temperature. This can be useful for a preliminary determination of the magnitude of the thermal expansion coefficients with their high magnetostriction and domain structures, in Invar alloy application areas.

Author Contributions: L.H. conceived and designed the experiments; L.H., T.G. and Y.Z. performed all the experiments of samples and wrote the manuscript; D.H. and Y.G. provided the sample materials. C.S. and F.P. analyzed the data, contributed scholarly through brainstorming sessions about functional properties. All authors have read and agreed to the published version of the manuscript.

Funding: This research was supported by the development of invar alloy Ni36 technology for aircraft manufacturing (grant No. 20201101011).

Institutional Review Board Statement: Not applicable.

Informed Consent Statement: Not applicable.

Data Availability Statement: The data presented in this study are available on request from the corresponding author.

Conflicts of Interest: The authors declare no conflict of interest.

\section{References}

1. Guillaume, C.E. Invar and its applications. Nature 1904, 71, 134-139. [CrossRef]

2. Sahoo, A.; Medicherla, V.R.R. Fe-Ni Invar alloys: A review. Mater. Today Proc. 2021, 43, 2242-2244. [CrossRef]

3. Yuan, J.P.; Yi, D.Q.; Yu, Z.M.; Huang, Z.C.; Wu, B.T.; Zhang, X. Influence of the deformation and heat treatment on the microstructures and properties of Invar alloy. Heat Treat. Met. 2005, 30, 50-53.

4. Liu, J. Application and development of low expansion alloys. Met. Funct. Mater. 2007, 14, 33-37.

5. Iwase, A.; Hamatani, Y.; Mukomoto, Y.; Ishikawa, N.; Chimi, Y.; Kambara, T.; Muller, C.; Neumann, R.; Ono, F. Anomalous shift of Curie temperature in iron-nickel Invar alloys by high-energy heavy ion irradiation. Nucl. Instrum. Methods B 2003, 209, 323-328. [CrossRef]

6. Matsushima, Y.; Sun, N.Q.; Kanamitsu, H.; Matsushita, M.; Iwase, A.; Chimi, Y.; Ishikawa, N.; Kambara, T.; Ono, F. Pressure dependence of the irradiation-induced ferromagnetism in Fe-Ni invar alloys. J. Magn. Magn. Mater. 2006, 298, 14-18. [CrossRef]

7. Goria, P.; Martinez-Blanco, D.; Blanco, J.A.; Smith, R.I. Neutron powder thermo-diffraction in mechanically alloyed $\mathrm{Fe}_{64} \mathrm{Ni}_{36}$ invar alloy. J. Alloy. Compd. 2010, 495, 495-498. [CrossRef]

8. Nickel and Its Uses; Nickel Magazine, 2011; Volume 26. Available online: https://nickelinstitute.org/nickel-magazine/nickelmagazine-vol-26-no-1-2011/ (accessed on 9 December 2021).

9. Shiga, M. Invar alloys. Curr. Opin. Solid State Mater. 1996, 1, 340-348. [CrossRef]

10. Matsushita, M.; Endo, S.; Miura, K.; Ono, F. Pressure induced magnetic phase transition in Fe-Ni Invar alloy. J. Magn. Magn. Mater. 2003, 265, 352-356. [CrossRef]

11. Vinogradov, A.; Hashimoto, S.; Kopylov, V.I. Enhanced strength and fatigue life of ultra-fine grain Fe-36Ni Invar alloy. Mater. Sci. Eng. A 2003, 355, 277-285. [CrossRef]

12. Pan, T.; Zhu, J.; Su, H.; Yang, C.F. Ni segregation and thermal stability of reversed austenite in a Fe-Ni alloy processed by QLT heat treatment. Rare Met. 2015, 34, 776-782. [CrossRef]

13. Chen, L.; Zhang, J.F.; Zhang, L.; Meng, L. Textures of high-strength and low-expansion Fe-Ni alloy wires during cold-drawing processes. Int. J. Miner. Metall. Mater. 2009, 16, 667-671.

14. James, J.D.; Spittle, J.A.; Brown, S.G.R.; Evans, R.W. A review of measurement techniques for the thermal expansion coefficient of metals and alloys at elevated temperatures. Meas. Sci. Technol. 2001, 12, R1-R15. [CrossRef]

15. Homenko, O.A. Origin and specific features of invar anomalies of physical properties: Fe-Ni alloys with an FCC lattice. Phys. Met. Metallogr. 2007, 104, 146-156. [CrossRef] 
16. Miodownik, A.P. The Invar behaviour of iron-nickel-platinum alloys. J. Magn. Magn. Mater. 1979, 10, 126-135. [CrossRef]

17. Shimitsu, M. Magnetovolume effects in itinerant electron ferromagnets. J. Magn. Magn. Mater. 1980, $20,47-55$.

18. Ducki, K.J. Structure and Precipitation Strengthening in a High Tempering Fe-Ni Alloy. Arch. Mater. Sci. Eng. 2007, 28, 203-210.

19. Van Schilfgaarde, M.; Abrikosov, I.A.; Johansson, B. Origin of the Invar effect in iron-nickel alloys. Nature 1999, 400, 46-49. [CrossRef]

20. Jiang, X.; Li, L.; Zhai, Q. Effect of annealing process on microstructure and properties of hot-rolled plate of Fe-36Ni invar alloy. Shanghai Met. 2012, 34, 14-18.

21. Muraoka, Y.; Okuda, H.; Shiga, M.; Nakamura, Y. Magnetic Properties and Magnetovolume Effects in GdxY1-xCo2 (x $\leq$ 0.2) Cluster Glasses. J. Phys. Soc. Jpn. 1981, 50, 2573. [CrossRef]

22. Dai, D.S.; Qian, K.M. (Eds.) Ferromagnetism, Science Press (Version 2) Handbook; Science Press: Beijing, China, 2017 ; ISBN 9787030530394. 